\title{
SPINAL ANAESTHESIA FOR TRANS-URETHRAL RESECTION OF BLADDER TUMOR IN A PATIENT WITH RECENT MYOCARDIAL INFARCTION
}

\author{
M. S. Raghuraman, A. Umeshkumar, V. R. Hemanth Kumar
}

1. Professor, Department of Anesthesiology. Mahatma Gandhi Medical College \& Research Institute, Pondicherry.

2. Post Graduate, Department of Anesthesiology. Mahatma Gandhi Medical College \& Research Institute, Pondicherry.

3. Assistant Professor, Department of Anesthesiology. Mahatma Gandhi Medical College \& Research Institute, Pondicherry.

\section{CORRESPONDING AUTHOR}

M. S. Raghuraman,

49 \& 50, Padmavathy Nagar Ext,

Kondur post, Cuddalore,

Tamil Nadu.

E-mail: rad_vig04@yahoo.co.in

Ph: 00919486837256

ABSTRACT: A 60 year old man with recent Inferior wall Myocardial infarction presented with haematuria and burning micturition after 2 days of admission to our hospital. Urologist was consulted and growth on the left lateral wall of bladder with clots found in the ultra-sound. Transurethral resection of bladder tumor was recommended immediately to control the bleeding and also to stabilize the patient. We know that administering Anaesthesia for a non cardiac surgery who had a recent myocardial infarction poses a higher risk of complications. We present our uneventful experience of spinal Anaesthesia in this patient who had undergone transurethral resection of bleeding bladder tumor successfully.

KEYWORDS: Anaesthesia Techniques-subarachnoid, Heart-myocardial function, Heartischemia

INTRODUCTION: Patients with known coronary artery disease exposed to anesthesia for non cardiac surgery have an increased morbidity and mortality when compared to normal population. In particular, administering anaesthesia for one, who had a recent myocardial infarction poses a higher risk of complications. The incidence of perioperative myocardial reinfarction in a patient who had a recent myocardial infarction undergoing non cardiac surgery is very high. $[1,2,3,4]$ we present our uneventful experience of spinal Anaesthesia in a 60 year old male for transurethral resection of bleeding bladder tumor who had myocardial infarction just 15 days before the surgery.

CASE REPORT: A 60 year old gentleman who is a chronic smoker and alcoholic presented with the complaints of breathlessness for 5 days. Past history revealed that he is a case of undiagnosed systemic hypertension and coronary artery disease who suffered an Inferior wall Myocardial infarction a week back and thrombolysed with Injection streptokinase at another Institute. 
On admission to our Institute, his general condition was stable, pulse 88/minute and regular, Blood pressure 100/70mmh. He looked pale but not cyanosed or icteric. Cardiovascular, respiratory, abdominal, and central nervous system examinations were unremarkable. Basic laboratory investigations were Haemoglobin=6.4 gm\%, Random Blood Sugar =101 mgs\%, urine albumin and sugar nil. Chest $\mathrm{X}$ ray revealed a normal study. Electrocardiogram revealed non significant $Q$ waves in lead III. 2 dimensional Echocardiography revealed regional wall motion abnormality, mild mitral regurgitation, and Ejection fraction $60 \%$. Patient was admitted in the cardiology unit with the following treatment, Tab. Isosorbide mononitrate 20mg BD, Tab. Pantoprazol 40 mg BD Tab. Metoprolol 25 mg BD, Tab. Atorvastatin $40 \mathrm{mg}$ OD, Tab. Aspirin $150 \mathrm{mg}$ OD and was under observation. After 2 days of admission the patient presented with haematuria and burning micturition, for which Urologist opinion was obtained. Ultrasonogram revealed a $5 \times 5 \mathrm{~cm}$ growth on the left lateral wall of bladder with clots in the bladder. Urologist recommended a cystoscopy with cauterization of bleeding point / transurethral resection of bladder tumor to control the bleeding and to stabilize the patient. Coagulation profile showed Bleeding time as 3 minutes, clotting time 6 minutes 30 seconds and platelet count was 450,000. Prothrombin time and activated partial thromboplastin time were within normal limits.

Two units of packed cells were transfused. Patient was accepted for Anaesthesia under American Society of Anesthesiologists class 3 and informed consent obtained with a plan of Spinal Anaesthesia plus obturator nerve block. Patient was nil per oral from the previous night and Tab. Ranitidine $150 \mathrm{mg}+$ Tab. Metoclopramide $10 \mathrm{mg}$ + Tab. Metoprolol $25 \mathrm{mg}$ were given at 7 A.M. on the day of surgery. Prior to Anaesthesia his vitals were blood pressure 116/80 $\mathrm{mmHg}$, Pulse rate $76 /$ minute and oxygen saturation was $100 \%$ on room air. Pre-loading was done with $500 \mathrm{ml}$ of $6 \%$ hydroxyl ethyl starch. Inj. Midazolam $1.5 \mathrm{mg}$ was given intra-venously 5 minutes before wheeling the patient to the Operation theatre.

PROCEDURE: Under strict aseptic precautions, spinal anaesthesia was given at L3-4 space, with $2.8 \mathrm{ml}$ which is a combination of a $2.2 \mathrm{ml}$ hyperbaric $0.5 \%$ Bupivacaine plus $0.6 \mathrm{ml}$ fentanyl . Obturator nerve block was given with $23 \mathrm{G}$ spinal needle after lithotomy position. The sensory level of blockade was up to T8. Intraoperatively 0.5 liter isotonic saline was administered. The patient was haemodynamically stable throughout the procedure which lasted for an hour and five minutes. Patient was shifted to recovery room and one unit of packed cells transfused after that he was transferred to intensive care unit. Lactated Ringers solution was given at 100 $\mathrm{ml} /$ hour for four hours, after that oral feeds started. Postoperative analgesia was provided with inj.morphine i.v. $8^{\text {th }}$ hourly for 24 hours. 12 lead ECG was taken twice daily for three days to rule out any fresh changes, in addition to the continuous ECG monitoring in intensive care unit. After a week patient was discharged and was doing well for three months of our follow-up.

DISCUSSION: In general, elderly patients have changes in their cardiovascular physiology because of aging and their coexisting diseases which makes them vulnerable to the haemodynamic instability. The anaesthetic management in the patients with the coronary artery disease should be focused on modulation of sympathetic nervous system responses and in controlling hemodynamic variables:[5]Maintaining the balance between myocardial oxygen requirements and myocardial oxygen delivery is crucial in preventing the myocardial ischemia. Any circumstances associated with tachycardia, systolic hypertension, diastolic hypotension and hypoxemia can unfavorably change this balance. Spinal Anaesthesia is preferred over 
general Anaesthesia in the elderly patients because of their hemodynamic benefits which includes a minimum decrease in myocardial contractility and a modest decrease in blood pressure and cardiac output. [6,7]

Elective surgery and Anaesthesia following myocardial infarction should be postponed for a certain times. Few studies recommend that the surgery should be postponed until the end of the healing stage of the myocardium which according to them is 3 months. ${ }^{[8,9]}$ It takes about 2 months for most of the infarctions to establish the vascularized scar tissue and one more month to consolidate. [9]

The type of surgery we encountered here is a semi emergency situation as the persistent bleeding from the tumor would eventually lead to severe anaemia which would worsen his already existing anaemic status ultimately affecting his cardiovascular status. So we decided to take him for the surgery and in this case we didn't go for General Anaesthesia, as we know that different inhalational and intravenous agents have different effects on the hemodynamics of the heart such as depression in myocardial contractility, reduction or increase in the heart rate and blood pressure which in our case would be detrimental. We could have opted for continuous epidural analgesia but for the following reasons 1) Shorter duration of the procedure done through the natural orifice.2) Chances for development of haematoma. 3) Starting antiplatelet/anti-coagulant drugs in the post-op period if necessary. We planned to use Lowmolecular weight heparin or tissue plasminogen activators with consultation of cardiologist, if the patient developed re-infarct in the peri-op period. We chose spinal Anaesthesia in our patient because it produces satisfactory surgical analgesia, causes minimal hemodynamic changes in this age group, avoids tracheal intubation and maintains spontaneous ventilation. Obturator block was added to avoid adductor contractions which otherwise would cause discomfort to the surgeon. ECG monitoring was done with lead II( for inferior wall) and $\mathrm{V}_{5}$ ( for lateral wall) to detect any changes in ST-T segment during peri-op period. In our patient, hemodynamic stability was maintained throughout the procedure because of addition of intrathecal fentanyl with reduction in local anaesthetic. We used $0.6 \mathrm{ml}$ ( $30 \mathrm{mcg}$ ) of fentanyl with $2.2 \mathrm{ml}$ of hyperbaric $0.5 \%$ bupivacaine thus reducing the dose of local anaesthetic which is responsible for the sympathetic blockade and hypotension. It also increases the onset and prolongs the duration of blockade. ${ }^{[10]}$

CONCLUSION: Semi-Emergency procedures like in our case (Bleeding Bladder Tumor) may be taken up for Anaesthesia and Surgery even in the case of recent M.I. Cardiac and haemodynamic status can be the major factors in determining the Anaesthesia technique and monitoring. Spinal Anaesthesia (preferably local anaesthetic plus fentanyl combination) can be safely administered in this set of patients, provided the cardiac and haemodynamic status are reasonably good, although this may need confirmation by further studies.

\section{REFERENCES:}

1. Tarhan S,Moffitt EA,Taylor WF,Giuliani ER. Myocardial infarction after general Anaesthesia. Anesth Analg 1977; 56: 455-461.

2. Steen PA, Tinker JH, Tarhan S. Myocardial reinfarction after Anaesthesia and surgery. JAMA 1978; 239:2 566-2570.

3. Rao TL, Jacobs KH, EL-Etr AA. Reinfarction following Anaesthesia in patients with myocardial infarction. Anesthesiology 1983;59:499-505. 
4. Shah KB, Kleinman BS, Sami H. Reevaluation of perioperative myocardial infarction in patients with prior myocardial infarction undergoing noncardiac operations. Anesth Analg 1990;71:231-235.

5. Mangano DT, Goldman L. Preoperative assessment of patients with known or suspected coronary disease. N Eng J Med 1995;333: 1750-1756.

6. G. Alec Rooke, Peter R. Freund, Arnold F. Jacobson. Hemodynamic response and change in organ blood volume during spinal anaesthesia in elderly men with cardiac disease. Anesth Analg1997; 85:99-105.

7. Mark JB, Steele SM. Cardiovascular effects of spinal Anaesthesia. Int Anesthesiol Clin 1989; 27:31-9.

8. Fraser,J.G, Ramachandran,P.R, Davis,H.S. Anaesthesia and recent myocardial infarction.J.Amer.med.Ass.1967;199:318.

9. Mallory G.K, White,P.D, Salcedo-Salcar,J. Speed of healing of myocardial infarction. Aner.Heart J.1939; 18: 647.

10. Kristiina S. Kuusniemi, Kalevi K. Pihlajama, Mikko T. Pitka"nen, Hans Y. Helenius, Olli A. Kirvela, MD, PhD. The Use of Bupivacaine and Fentanyl for Spinal Anesthesia for Urologic Surgery. Anesth Analg 2000; 91:1452-6. 